\title{
Commentary: Target hemodynamic goals after cardiac surgery-Time for a paradigm shift?
}

Juan N. Pulido, MD

\footnotetext{
From the Cardiovascular Intensive Care Unit, Department of Cardiothoracic Anesthesiology and Critical Care Medicine, Swedish Heart and Vascular Institute, Swedish Medical Center, US Anesthesia Partners Washington, Seattle, Wash.

Disclosures: Author has nothing to disclose with regard to commercial support.

Received for publication Feb 8, 2019; accepted for publication Feb 11, 2019; available ahead of print March 21, 2019.

Address for reprints: Juan N. Pulido, MD, Cardiothoracic Anesthesiology and Critical Care Medicine, Swedish Heart and Vascular Institute, Swedish Medical Center, US Anesthesia Partners Washington, 550 17th Ave, No.680, Seattle, WA 98122 (E-mail: juan.pulido@swedish.org).

J Thorac Cardiovasc Surg 2019;158:1382-3

$0022-5223 / \$ 36.00$

Copyright (c) 2019 by The American Association for Thoracic Surgery

https://doi.org/10.1016/j.jtcvs.2019.02.050
}

As part of routine postoperative cardiac surgical critical care, target hemodynamic parameters are set for vasoactive drip titration, volume administration, or diuresis. These goals are usually empirically based on institutional or surgeon preference and experience, with the intention of preventing bleeding from surgical sites while maintaining adequate organ perfusion.

Despite the lack of good data to guide blood pressure management after cardiac surgery and the understanding that blood pressure may not necessary correlate with perfusion, it has been generally accepted that aiming for a mean arterial pressure (MAP) of 60 to $90 \mathrm{~mm} \mathrm{Hg}$ and a systolic blood pressure (SBP) of 90 to $140 \mathrm{~mm} \mathrm{Hg}$ are reasonable goals. ${ }^{1}$ It is well known, however, that patients with chronic hypertension may require higher blood pressure for optimal organ perfusion in the acute setting, in particular when it comes to renal and cerebral perfusion. Because hypertension is extremely common in cardiac surgical patients, and stroke and acute kidney injury both have profound prognostic implications, optimal blood pressure parameters are sometimes sacrificed in high-risk patients to avoid bleeding. The importance of individualized blood pressure management in perioperative care is being studied in noncardiac surgery, and recent data suggest a reduction in postoperative organ dysfunction with this approach.

In this issue of the Journal, McIlroy and colleagues ${ }^{3}$ explore the association of postoperative blood pressure and bleeding after cardiac surgery. This study is a preplanned secondary analysis of a recent randomized controlled trial, the LICRA (Limiting IV chloride to Reduce AKI) trial, ${ }^{4}$ the aim of which was to determine whether a comprehensive perioperative protocol for administration of chloride limiting intravenous fluid administration would reduce perioperative acute kidney injury in cardiac surgical patients at a single institution. Univariate and multivariate regression analyses of 793 cardiac surgical patients $1.28 ; P=.01)$.

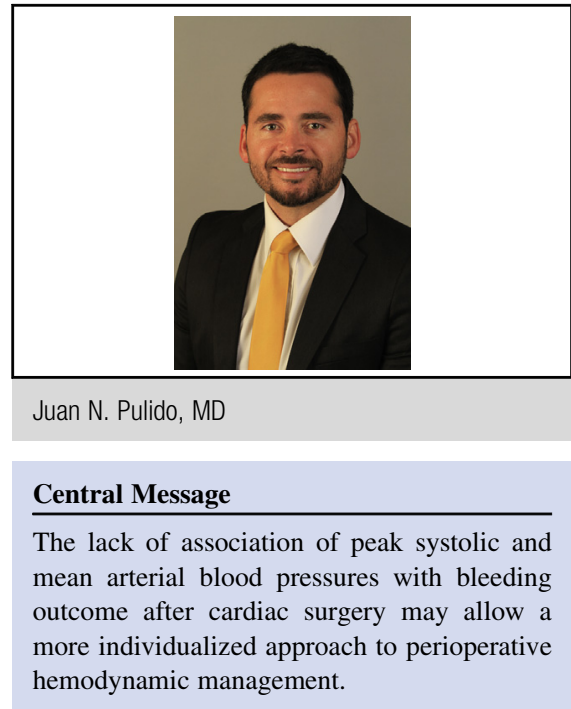

See Article page 1370.

explored the association of peak SBP, peak MAP, and peak central venous pressure (CVP) recorded postoperatively on the day of surgery with multiple metrics of bleeding, including chest tube drainage (CTD) on postoperative day 0 , postoperative red blood cell transfusion, and unplanned surgical reexploration for bleeding before the completion of postoperative day 1 .

The analysis excluded patients with preexisting coagulopathy, thrombocytopenia, or recent clopidogrel or anticoagulant administration. The chest tube management and transfusion trigger was standard and relatively well controlled. Exploration of the relationships of peak SBP, MAP, and CVP each with the primary outcome of CTD (in milliliters per hour) did not support a clear inflection point; on the basis of that exploration, hemodynamic parameters were included as linear terms in models. Some interesting findings included that higher peak MAP was associated with reduced likelihood of transfusion (odds ratio, $0.95 ; 95 \%$ confidence interval, $0.93-0.97 ; P \leq .001)$ and in-hospital mortality (odds ratio, $0.91 ; 95 \%$ confidence interval, 0.84-0.99; $P \leq .03$ ), whereas increasing peak CVP was associated with increased likelihood of surgical reexploration (odds ratio, 1.16; 95\% confidence interval, 1.04-

McIlroy and colleagues ${ }^{3}$ went further to explore an effect of hemodynamic outliers on bleeding by dichotomizing 
each hemodynamic parameter at the upper decile (peak SBP $>145 \mathrm{~mm} \mathrm{Hg}, \mathrm{MAP}>95 \mathrm{~mm} \mathrm{Hg}$, and CVP >16 mm Hg) and did not find an association between upper decile peak SBP or MAP and increased CTD, transfusion, or surgical reexploration. Nevertheless, the upper decile of CVP was associated with increased CTD, transfusion, surgical reexploration, and mortality.

This study provides refreshing new evidence and at the least is thought provoking regarding a paradigm shift in perioperative cardiac surgical care, as there was a lack of association of blood pressure and bleeding. Moreover, the association of high CVP with bleeding should also be acknowledged and investigated, because it is likely more important when it comes to the need for reexploration. These findings are welcome and would allow further study of the effect of individualized blood pressure management after cardiac surgery, provide hope for the reduction of $\mathrm{AKI}$ and stroke, complications that have true prognostic implications and perhaps could be prevented by more liberal blood pressure targets.
Despite these findings, it is important to underline the "art" of surgery and such aspects as intraoperative tissue frailty. These are variables that never would be possible to account for objectively in a study and are of crucial importance, emphasizing the need for a detailed handoff between the surgical team and the critical care team.

\section{References}

1. Stephens RC, Whitman GJ. Postoperative critical care of the adult cardiac surgical patient. Part I: routine postoperative care. Crit Care Med. 2015;43:1477-97.

2. Futier E, Lefrant JY, Guinot PG, Godet T, Lorne E, Cuvillon P, et al: INPRESS Study Group. Effect of individualized vs. standard blood pressure management strategies on postoperative organ dysfunction among high-risk patients undergoing major surgery: a randomized clinical trial. JAMA. 2017; 318:1346-57.

3. McIlroy D, Murphy D, Kasza J, Bhatia D, Marasco S. Association of postoperative blood pressure and bleeding after cardiac surgery. J Thorac Cardiovasc Surg. 2019;158:1370-9.e6.

4. McIlroy D, Murphy D, Kasza J, Bhatia D, Wutzlhofer L, Marasco S. Effects of restricting perioperative use of intravenous chloride on kidney injury in patients undergoing cardiac surgery: the LICRA pragmatic controlled clinical trial. Intensive Care Med. 2017;43:795-806. 